\title{
Vasodilator-stimulated phosphoprotein regulates osteosarcoma cell migration
}

\author{
GANG WU ${ }^{1,3^{*}}$, LEI WEI ${ }^{2 *}$, AIXI YU ${ }^{1}$, MING ZHANG ${ }^{3}$, \\ BAIWEN QI ${ }^{1}, \mathrm{KE} \mathrm{SU}^{2}$, XIANG HU ${ }^{1}$ and JING WANG ${ }^{2}$ \\ ${ }^{1}$ Department of Orthopedics, Zhongnan Hospital of Wuhan University, Wuhan, Hubei 430071; \\ ${ }^{2}$ Department of Pathology and Pathophysiology, Medical School of Wuhan University, Wuhan, \\ Hubei 430071, P.R. China; ${ }^{3}$ Department of Molecular Pharmacology and Biological Chemistry, \\ Feinberg School of Medicine, Northwestern University, Chicago, IL 60611, USA
}

Received July 13, 2011; Accepted August 12, 2011

DOI: $10.3892 /$ or.2011.1438

\begin{abstract}
Vasodilator-stimulated phosphoprotein (VASP) has been reported to play an important role in the process of cell migration and tumor metastasis. However, to date, no study has examined VASP expression and its function in osteocarcoma cells. In this study, we analyzed the effect of VASP on osteosarcoma cell migration and the signal transduction pathways involved. We used two osteosarcoma cell strains (Mg-63 and Saos-2 cells) with different metastatic potential. Silencing of VASP gene expression was carried out using RNA interference in these cells. Knockdown of expression at the transcriptional or translational level was determined by RT-PCR or Western blot analysis, respectively. The metastatic potential of the tumor cells was determined by a wound healing migration assay. VASP mRNA expression was also determined in 30 human osteosarcoma samples. Furthermore, Rac1 was determined as a regulator of VASP function. RT-PCR and Western blotting showed that Mg-63 cells had a significantly higher VASP expression at both the transcriptional and translational levels compared to Saos- 2 cells. The wound healing assay revealed that $\mathrm{Mg}-63$ cells had more migratory potential compared to Saos-2 cells. The effect was found to be reversible when VASP was knocked down by siRNA in Mg-63 cells. Specimens from human patients with metastases had higher VASP expression compared to specimens of patients without metastases. Knockdown of Racl resulted in inhibition of VASP expression in sarcoma cells. These results suggest that VASP
\end{abstract}

Correspondence to: Dr Aixi Yu, Department of Orthopedics, Zhongnan Hospital of Wuhan University, 169 Donghu Road, Wuhan, Hubei 430071, P.R. China

E-mail: yuaixi666@gmail.com

*Contributed equally

Key words: vasodilator-stimulated phosphoprotein, osteosarcoma, metastasis, cell migration, Rac1 protein regulates osteosarcoma cell migration and metastasis. Rac1 and VASP interaction may be a potential target for osteosarcoma treatment.

\section{Introduction}

Osteosarcoma is the most prevalent primary malignant bone tumor in children and young adults. Although the life expectancy of osteosarcoma patients has been prolonged due to improvement of neoadjuvant chemotherapy and surgical resection during the past three decades, the long-term survival and quality of life is still unsatisfactory. Poor outcome of osteosarcoma is mainly caused by metastatic disease, and previous studies have demonstrated that more than $30 \%$ of patients already display distant metastasis without clinical detectable signs at the time of diagnosis $(1,2)$.

Vasodilator-stimulated phosphoprotein (VASP), a member of the Ena/VASP family, has been implicated in regulating key cellular functions, such as shape change, adhesion and migration, which is mainly due to its ability to modify dynamic action cytoskeleton (3). In addition to its role in normal cell growth (4,5), embryonic development (6) and homeostasis (7), VASP plays an essential role in many diseases, such as cancer metastasis $(8,9)$, thrombosis $(10)$, arteriosclerosis (11) and nephritis (12).

Expression of VASP has been reported to be dysregulated in several types of malignancies and is relevant to tumor metastasis. For example, its level was found to be significantly increased in lung adenocarcinomas and gastric carcinomas compared to normal tissue, and this increase was correlated with the metastatic ability of these tumors $(8,9)$. Depletion of VASP in breast cancer MDA-MB-231 cells resulted in decreased cell migration (13) and, conversely, enhanced VASP in breast cancer MDA-231 cells led to increased cell mobility (14). These findings suggest that VASP acts as a positive regulator of cell motility and tumor metastasis. However, there is a debate concerning its role in cell migration. When VASP was overexpressed in fibroblasts, cell mobility was decreased in a dose-dependent manner, while depletion of VASP enhanced cell movement $(15,16)$. Thus, how VASP affects cell mobility 
and the related underlying mechanism of its action are not fully known.

Rac1, a member of the Rho family of small GTPase, is also implicated in the processes of cell migration, cytoskeleton reorganization and formation of lamellipodia. Activation of Rac has been demonstrated to play an important role in processes induced by growth factor (17) and integrin (18). During cell migration, Rac1 translocates to the plasma membrane (19), interacts with WAVE via an adaptor protein, the insulin receptor substrate Irsp53, and then stimulates Arp2/3-mediated actin polymerization (20).

Since previous studies suggest that VASP plays an essential role in the process of cell migration and tumor metastasis, we reasoned that it is valuable to explore the role of VASP in osteosarcoma since no studies to date have been carried out in both osteosarcoma cells in vitro or human tissues in vivo. Here, we analyzed VASP expression in different osteosarcoma cell lines and human clinical samples. Furthermore, we demonstrated that the silencing of VASP affects osteosarcoma cell migration and tumor metastasis, and this action involves the small GTPase Rac1 signal transduction pathway.

\section{Materials and methods}

Cell culture and transfection. In the present study we used human osteosarcoma Mg-63 cells (characterized by a high metastatic potential in vivo) and Soas- 2 cells (characterized by a low metastatic potential) $(21,22)$. These two cell lines were purchased from ATCC (LGC Promochem, Wesel, Germany), and were cultured in DMEM medium (Sigma, USA) supplemented with $10 \%$ FBS (Amresco, USA) and 1\% penicillin/ streptomycin.

For transient transfection, the Rac1-directed siRNA vector was transfected using Lipofectamine 2000 (Invitrogen, USA) as specified in the manufacturer's instructions. Racl was silenced using specific siRNA (synthesized by Bibobio Co., China) which are as follows: sense 5'-UGAAGAAGAGGAAGAGAAAdTdT-3' and anti-sense 3'-dTdTACUUCUUCUCCUUCUCUUU-5'. For establishing stable clones of the VASP-siRNA plasmid and empty vector, VASP-siRNA and vector were transfected using Lipofectamine 2000. Stable clones were selected using blasticidin at $5 \mu \mathrm{g} / \mathrm{ml}$ for 3 weeks.

Tissue specimens. We recruited 44 human patients diagnosed with osteosarcoma. The study was carried out according to the Chinese National Ethical Guidelines ('Code for Proper Secondary Use of Human Tissue', Chinese Federation of Medical Scientific Societies). A written informed consent was obtained from each subject prior to enrollment in the study. The detailed clinical data were described in our previous study (23). Tissues were stored at $-80^{\circ} \mathrm{C}$ until further use. Specimens were dissected into samples $0.5 \mathrm{~mm}$ in diameter for RNA isolation for the RT-PCR analysis.

Wound healing assay. A wound healing assay was used to determine the migratory potential of the cells. For this study, $5 \times 10^{5}$ cells were plated into each well of a 6 -well plate overnight. On the following day, cells were serum-starved and parallel wounds were made using a $1000-\mu 1$ pipette tip. Images were collected at various times for the same spots. The cell migration distance was evaluated by measuring the width of the wound divided by two and subtracting this value from the initial half-width of the wound. Average velocity was calculated based on different observation times.

Isolation of RNA and RT-PCR. Total RNA was extracted from cells or tumor specimens using the TRIzol reagent (Invitrogen, USA). First-strand complementary DNA (cDNA) was synthesized from total RNA in a final volume of $20 \mu \mathrm{l}$ using Superscript II (Invitrogen, USA). cDNA was amplified by PCR using specific primers corresponding to mRNA encoding human gene products. Anealing temperatures for VASP and Racl were 65 and $56^{\circ} \mathrm{C}$. Amplification products were resolved by electrophoresis on a $1.5 \% \mathrm{w} / \mathrm{v}$ agarose gel. Primers used are listed as follows: VASP, sense 5'-GCGCCTGGTACCATGA CGCAAGTTGGGGAGAAAACC-3' and antisense 5'-GCG CCTGGATCCCAGGGAGAACCCCGCTTCC-3'; Rac1, sense 5'-AGACGGAGCTGTAGGTAAAA-3' and antisense 5'-GCAGGACTCACAAGGGA-3'.

Protein extraction and Western blot analysis. For determining the translation expression of VASP or Rac1, cells were lysed in lysis buffer [50 mM Tris- $\mathrm{HCl}(\mathrm{pH} 7.4), 150 \mathrm{mM} \mathrm{NaCl}$, $1 \%$ Triton X-100, 0.25\% deoxycholate, $1.5 \mathrm{mM} \mathrm{MgCl}_{2}, 1 \mathrm{mM}$ EGTA, $1 \mathrm{mM}$ phenylmethylsulfonyl fluoride (PMSF), $10 \mathrm{mM}$ $\mathrm{NaF}, 10 \mathrm{mM}$ pervanadate, $10 \mu \mathrm{g} / \mathrm{ml}$ leupeptin and $10 \mu \mathrm{g} / \mathrm{ml}$ aprotinin], and the protein concentration in the cell lysates was measured using the BCA protein assay kit (Sangon Biotech, China). Equal amounts of protein were separated on $12 \%(\mathrm{v} / \mathrm{v})$ SDS-PAGE and transferred to a nitrocellulose membrane. After blocking with 5\% milk, the membrane was incubated with polyclonal mouse antibody against VASP (1:2000; Immuno Globe, Germany) or Rac1 (1:500; Cytoskeleton). After three washings, the membrane was incubated with horseradish peroxidase (HRP)-conjugated goat anti-mouse IgG (1:300; Santa Cruz Biotechnology) for $1 \mathrm{~h}$ at room temperature. The ECL immune enzymic reaction kit (Pierce Biotechnology) was used for immunodetection. The band intensity was analyzed using Quantity One software (Bio-Rad).

\section{Results}

Increased expression of VASP correlates with the migratory potential of cells. Cell migratory ability is considered to be well correlated with the metastatic potential of the cells and is one of the first steps in the process of tumor metastasis. Mg-63 cells had a much higher metastasis potential when compared to the Saos-2 cells as determined by the wound healing assay. Mg-63 cells showed a 3.8-fold higher migratory potential compared to the Soas- 2 cells, which was determined to be statistically significant $(\mathrm{p}<0.01)$ (Fig. 1).

We observed a significantly increased transcriptional level of VASP in the Mg-63 cells as compared to the Saos-2 cells $(\mathrm{p}<0.01)$ (Fig. 2A). Similarly, augmented VASP expression at the protein level was also observed in Mg-63 cells when compared to the Saos-2 cells using Western blot analysis (Fig. 2B and C).

Knockdown of VASP results in the down-regulation of migratory potential in osteosarcoma cells. In the literature, 


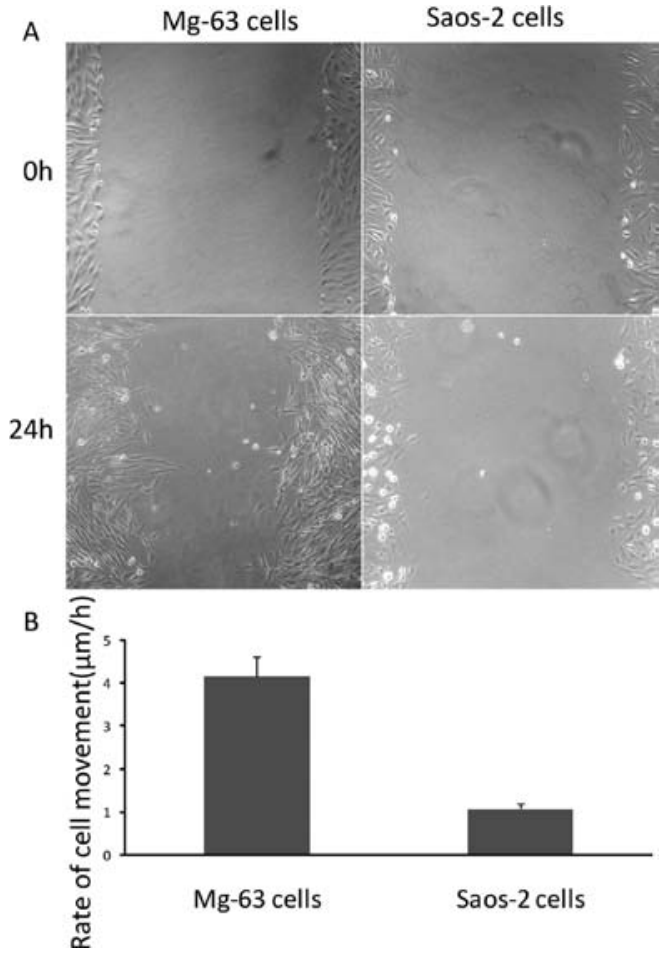

Figure 1. Rate of Mg-63 cell movement is higher than that of the Saos-2 cells. (A) After serum-starvation for $12 \mathrm{~h}$, a wound was made using a 1000- $\mu \mathrm{l}$ pipette tip. Images were captured $24 \mathrm{~h}$ after scratching; (B) Mg-63 cells moved faster than the Sao- 2 cells. The average velocity was calculated using the migration distance divided by the observation time $(24 \mathrm{~h})$. The difference between the velocities of these two cells was significant $(\mathrm{p}<0.01)$. Results are the mean $\pm \mathrm{SD}$ of six measurements at the same time.
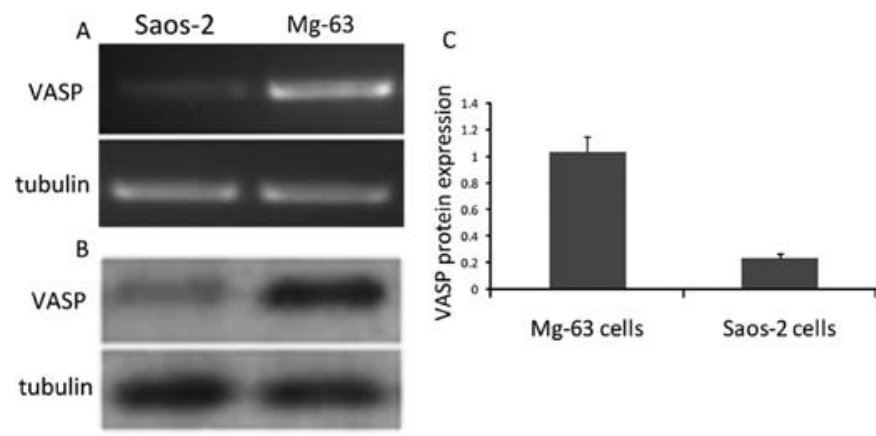

Figure 2. VASP expression in Mg-63 cells is higher than that in Saos-2 cells. (A) RT-PCR indicated that VASP mRNA was higher in the Mg-63 cells than that in the Saos-2 cells. Tubulin was used as a loading control. (B) VASP expression was confirmed by Western blot analysis. Tubulin was used as a loading control. (C) VASP protein in the $\mathrm{Mg}$-63 cells was significantly higher than that in the Saos- 2 cells $(\mathrm{p}<0.01)$. Shown are the mean values \pm SD of results from 4 independent experiments.

a positive correlation between cell migration/movement and expression of VASP has been documented (4,9,10,13-15). Our results are in line with these observations as we also observed a higher competency of cell migration and VASP expression in Mg-63. To gain more insight, we silenced the VASP using specific siRNA in the Mg-63 cells. Silencing of VASP in the Mg-63 cells was confirmed by RT-PCR and Western blot analysis (Fig. 3).

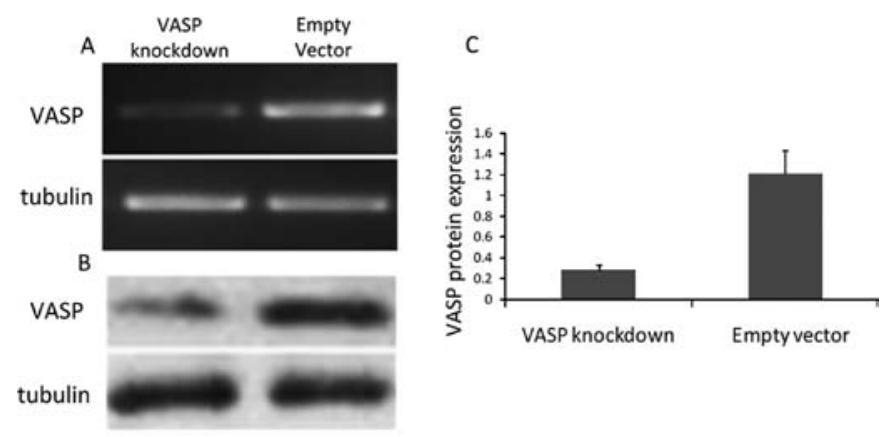

Figure 3. Characterization of siRNA-mediated VASP kockdown. (A) Two pools of stable clones of Mg-63 cells were established, VASP mRNA level was determined by RT-PCR. Tubulin was used as a loading control. (B) VASP protein expression was confirmed by Western blot analysis. Tubulin was used as a loading control. (C) Approximately $77 \%$ inhibition of the VASP protein was achieved by the VASP-siRNA plasmid compared to the empty vector $(\mathrm{p}<0.01)$. Bars indicate the mean $\pm \mathrm{SD}$ of triplicate experiments.

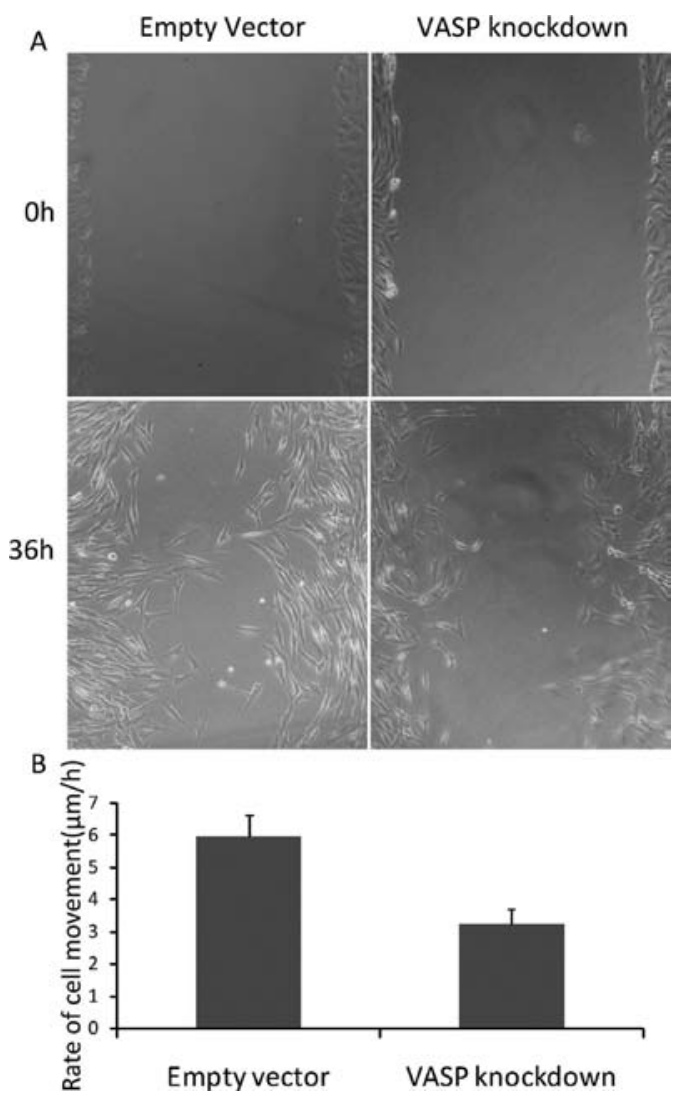

Figure 4. Depletion of VASP inhibits cell migration. (A) Two pools of stable clones were established using the VASP-siRNA plasmid and empty vector Cell mobility was determined using a wound healing assay. Images were captured $36 \mathrm{~h}$ after scratching. (B) VASP knockdown resulted in a decreased mobility compared to the empty vector group $(\mathrm{p}<0.01)$. Results are the mean \pm SD of six measurements at the same time.

After silencing of VASP in the Mg-63 cells, the effect on the migratory potential of these cells was determined using a wound healing assay at $36 \mathrm{~h}$. As depicted in Fig. 4, VASPsilenced Mg-63 cells lost their migratory capability by $55 \%$ when compared to the cells transfected with the empty vector alone. In contrast, the Saos-2 cells which had lower VASP expression than Mg-63 showed reduced cell migration. Taken 
Table I. Clinical features of the osteosarcoma cases.

\begin{tabular}{|c|c|c|}
\hline Features & Total no. & Percentage \\
\hline \multicolumn{3}{|l|}{ Age (years) } \\
\hline$<12$ & 1 & 3.33 \\
\hline $13-20$ & 11 & 36.67 \\
\hline $21-30$ & 8 & 26.67 \\
\hline $31-40$ & 8 & 26.67 \\
\hline$>41$ & 2 & 6.67 \\
\hline \multicolumn{3}{|l|}{ Gender } \\
\hline Male & 22 & 73.33 \\
\hline Female & 8 & 26.67 \\
\hline \multicolumn{3}{|c|}{ Location of the primary tumor } \\
\hline Distal femur & 9 & 30.00 \\
\hline Proximal tibia & 7 & 23.33 \\
\hline Humerus & 3 & 10.00 \\
\hline Tibia diaphysis & 2 & 6.67 \\
\hline Femur diaphysis & 7 & 23.33 \\
\hline Other & 2 & 6.67 \\
\hline \multicolumn{3}{|l|}{ Histological type } \\
\hline Osteoblastic & 18 & 60.00 \\
\hline Small cell & 1 & 3.33 \\
\hline Chondroblastic & 4 & 13.33 \\
\hline Round cell & 2 & 6.67 \\
\hline Fibroblastic & 4 & 13.33 \\
\hline Mixed & 1 & 3.33 \\
\hline \multicolumn{3}{|l|}{ Metastasis } \\
\hline No & 13 & 43.33 \\
\hline Lung & 15 & 50.00 \\
\hline Other & 2 & 6.67 \\
\hline
\end{tabular}

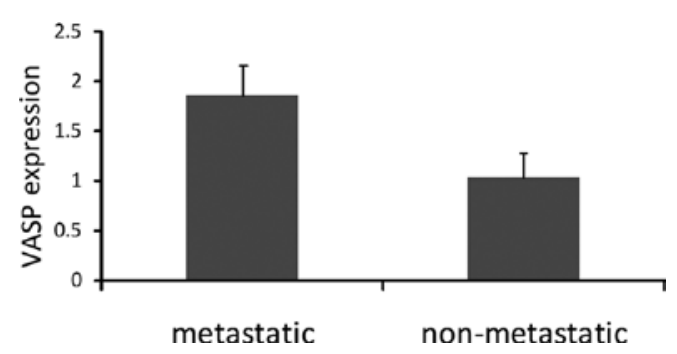

Figure 5. VASP mRNA is expressed higher in patients with metastasis than in patients without metastasis $(\mathrm{p}<0.01)$. Seventeen patients with distant metastasis and 13 without metastasis were analyzed by RT-PCR. Results are the mean $\pm \mathrm{SD}$ of samples.

together, these results suggest that VASP is at least partly responsible for regulating the motility of osteosarcoma cells.

Transcriptional (mRNA) expression of VASP in human tissue samples. We isolated RNA samples from 30 human osteosarcoma patients. Table I depicts the anthropometric and clinical features of the subjects recruited with osteosarcoma in the present study. These 30 subjects were divided into two groups

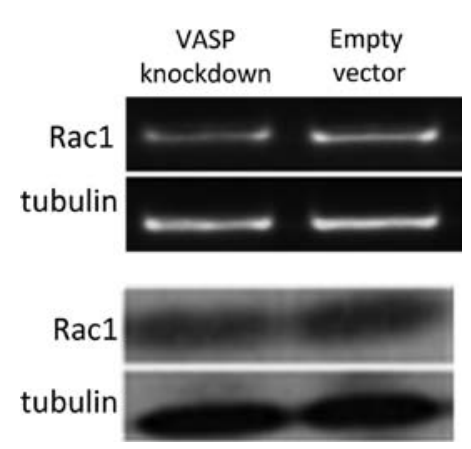

Figure 6. VASP depletion results in no detectable change in Rac1 expression. Racl expression was tested in the two established stable pools of Mg-63 cells. RT-PCR and Western blot analysis showed that depletion of VASP did not affect Rac1 expression. Tubulin was used as a loading control.

on the basis of the presence (Group I; n=17) and absence (Group II; $\mathrm{n}=13$ ) of metastasis.

We determined the mRNA expression of VASP in these tissue samples using semi-quantitative RT-PCR. A significantly increased mRNA expression was observed in Group I subjects compared to the subjects in Group II (p<0.01) (Fig. 5). Increased expression of VASP in subjects with metastasis suggests that VASP may be involved in regulating osteosarcoma metastasis in vivo.

Involvement of the Racl pathway. To determine whether there is any involvement of activation of the Racl signal transduction pathway in osteosarcoma cells, we determined the expression of Rac1 in VASP-silenced Mg-63 cells. We observed no significant difference in the expression of Racl both at the mRNA and protein level in the Mg-63 and VASP-silenced Mg-63 cells (Fig. 6).

We also examined expression of VASP in the Rac1-silenced Mg-63 cells. Silencing of Rac1 was carried out using Rac1directed siRNA and a negative siRNA control (as described in Materials and methods). Rac1-siRNA oligonucleotides demonstrated an efficient inhibitory effect on Rac1 expression both at the mRNA and protein level compared to the negative control (Fig. 7A and B). A significant decreased expression of VASP was found in the Racl-silenced cell lines when compared to the controls (Fig. 7). In addition, as depicted in Fig. 8, a significant inhibition in migration was observed in the Rac1-silenced Mg-63 cells compared to the control cells. Our results demonstrated that VASP moderated osteosarcoma cell migration via the Racl pathway and VASP may act as a downstream effector of Rac1.

\section{Discussion}

Cancer metastasis is a complicated process involving the invasion of the host matrix, intravasation and extravasation of blood or lymphatic vessels, and dissemination and proliferation in distant organs (24). Metastatic potential of a tumor is primarily dependent on the migratory ability of the cells $(25,26)$.

In this study, we demonstrated that VASP expression is associated with the metastatic potential of cells using two different cellular models of osteosarcoma. Mg-63 cells, which 

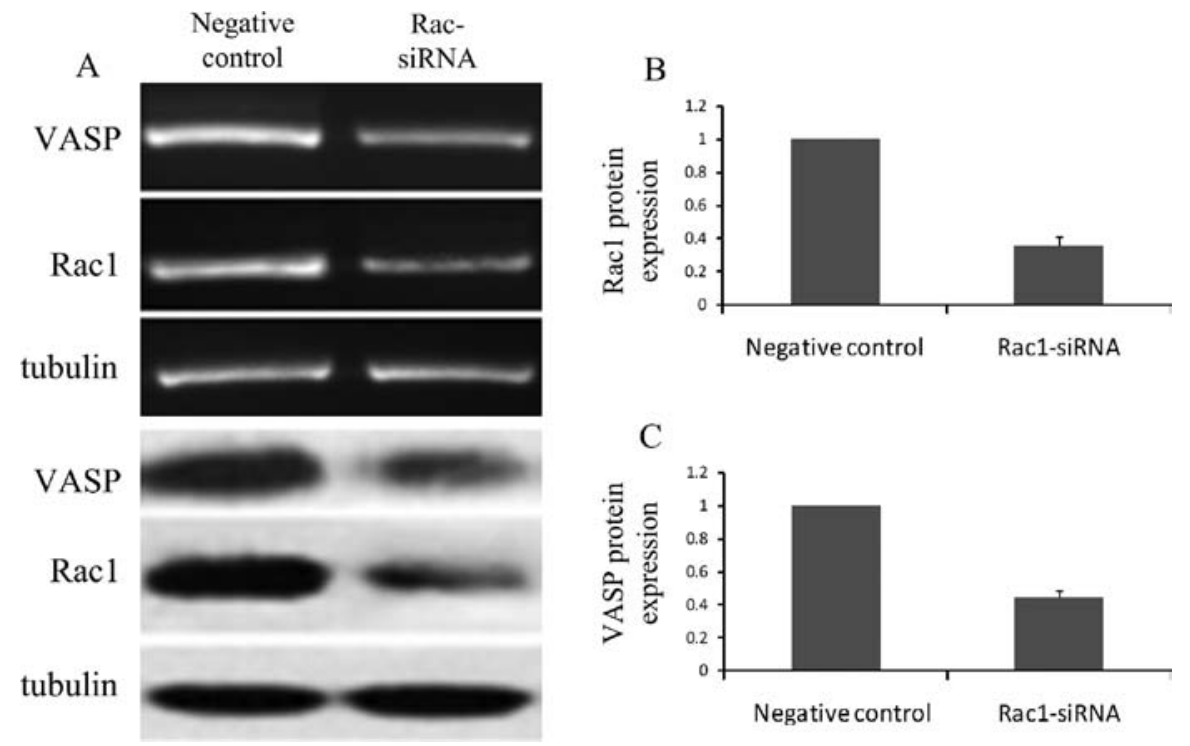

Figure 7. Depletion of Rac1 inhibits VASP expression. (A and B) Characterization of siRNA oligos against Rac1. Mg-63 cells were transiently transfected using Rac1-directed siRNA oligos and negative control oligos. Forty-eight hours after transfection, total mRNA or protein was isolated for analyzing Rac1 expression using RT-PCR or Western blot analysis. Expression level of Rac1 in Rac1-depleted cells was normalized to that in cells treated with negative siRNA control. Rac1-siRNA showed the effective inhibition of Racl in the Mg-63 cells, with a reduced Racl level (36\% to the control). Tubulin was used as a loading control. (A and C) Expression of VASP gradually decreased with depletion of Rac1. After cells were transfected with Rac1-siRNA, mRNA or protein was isolated for VASP expression. Rac1-siRNA resulted in a significant decrease in VASP.
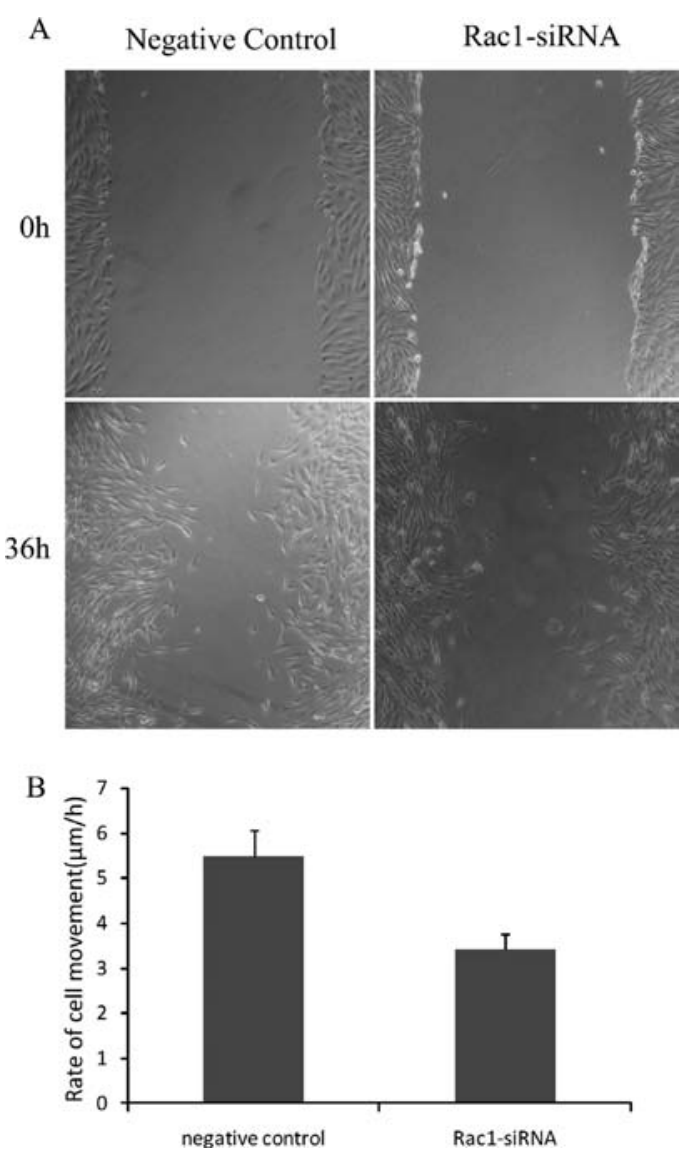

Figure 8. Depletion of Rac1 inhibits migration. (A) Mg-63 cells were transiently transfected using Racl-siRNA and negative siRNA, respectively. Twenty-four hours after transfection, cells were detached and plated into a 6 -well plate at a concentration of $5 \times 10^{5}$ cells/well. Twelve hours later, parallel wounds were made. Images were captured right after scratching or $36 \mathrm{~h}$ after scratching. (B) Rac1 knockdown resulted in a decreased mobility compared to the negative siRNA group $(\mathrm{p}<0.01)$. Results are the mean \pm SD of six measurements. expressed higher levels of VASP, moved much faster than Saos- 2 cells. Our observations are in accordance with various reports presented in the literature for other types of cancer cells $(8,9,13,14)$. Furthermore, we silenced VASP using specific siRNA in Mg-63 cells. We observed that silenced cells had a decreased migratory potential when compared to the control. Taken together, our data indicate that VASP may be a positive regulator of osteosarcoma cell migration.

We also determined VASP mRNA expression in tumors obtained from human osteosarcomas. We observed that all of the human osteosarcoma samples had VASP expression. However, a significant higher expression was observed in patients with metastasis. To our knowledge, this is the first report to demonstrated that the mRNA expression of VASP is correlated with the progression of osteosarcoma in patients. Thus, VASP may be a promising biomarker in determining metastasis of osteosarcoma.

In the process of cancer metastasis, VASP has been documented to act as an actin anti-capping protein $(27,28)$. This allows actin filament elongation and clustering at the leading edge toward the membrane, which can regulate formation of membrane protrusions such as lamellipodia and filopodia, consequently resulting in modified cell motility and tumor metastasis (3). Rottner et al demonstrated that, in a B16 melanoma cell line, VASP was localized in a line running along the tips of the protruding membrane and its expression was proportional to the protrusion rate (29). Depletion of VASP proteins in growth cones of neurons resulted in a reduction in long filaments, and an increase in short action filament has been demonstrated in another study (30). In contrast, Bear et al demonstrated that enrichment of VASP in fibroblasts promotes elongation of actin filament (16). In addition, VASP was able to counteract capping protein $(\mathrm{CP})$, delaying termination of actin filament 
elongation. In addition to their anti-capping ability, several other mechanisms exist by which VASP proteins regulate actin-based membrane protrusion, including depression of actin-filament branching (31) and recruitment of profiling (32).

As discussed above, the role of VASP proteins in facilitating actin-filament and membrane protrusions has been well-established. However, the global cell motility affected by VASP is not fully determined and seems to be cell-type dependent. In the present study, Mg-63 cells with higher expression of VASP had higher migratory potential as compared to Saos-2 cells. Inhibition of cell migration after silencing VASP in Mg-63 cells clearly suggests that VASP plays a positive role in regulating osteosarcoma cell motility. Our observations are in line with other studies carried on Listeria $(33,34)$, macrophages $(35)$, and several types of cancer cells $(8,9,14,36,37)$. However, there have been reports of an opposite effect of VASP. For example, studies on neurocytes (38), platelets (7) and fibroblasts (15) found that VASP negatively regulated cell migration. Since cell movement is the result of the co-ordination of four major processes which include extension of membrane protrusion, stabilization of protrusion by attachment via focal adhesion, translocation of the cell body and detachment of the rear region (39), this contradictory phenomenon implies that cell movement is a complicated process.

Rac1 is a member of the Rho family of small GTPase, which has been implicated in actin cytoskeleton organization in diverse cellular functions including cell motility. Rac1 is widely expressed in many tissues, while Rac2 and Rac3 were restrictly expressed in the hematopoietic and nervous systems, respectively (40). Rac1 was found to be required in growth factor- (17) and integrin-mediated (18) cytoskeleton remodeling and migration. Other studies in the literature also showed that Racl plays a crucial role in tumor cell migration $(9,13,36)$. Given the importance of both Racl and VASP in the actin-based process, we tried to determine whether there is a relationship between these two proteins.

The molecular mechanisms that lead to a harmonious interplay between VASP proteins and Rac proteins are poorly understood. Therefore, we silenced Rac1 in Mg-63 cells and observed a significant decrease in the expression of VASP both at the transcription and translational levels. In contrast, when VASP was silenced in Mg-63 cells, we did not observe any change in the expression of Racl either at the transcriptional or translational levels. These results clearly suggest that VASP may act as a downstream effector of Rac1.

One of the possible factors that may contribute to the cytoskeletal effect of VASP is serine/threonine p21-activated kinase (PAK). PAK was generally identified as a direct substrate of Rac. PAK comprises five proline-rich regions, while VASP contains an N-terminal Ena/VASP homology 1 (EVH1) domain which can bind to a specific proline-rich motif (41). After serine 21 phosphorylation of PAK by Rac, the association of VASP/PAK was found to be enhanced while Nck/PAK was inhibited (42). Consistent with our result here, in breast cancer cells, inhibition of Rac1 also decreased the expression of VASP, and cGMP-dependent protein kinase (PKG) was suspected to play a crucial role to bridge the relationship between Rac and VASP $(13,36,37)$. In a novel Rac/ PAK/GC/cGMP pathway, Rac, using its downstream effector PAK, was found to activate transmembrane guanylyl cyclases
(GCs), resulting in elevated cGMP. Then as a major target of cGMP, activated PKG regulated VASP via the cGMP/PKG/ VASP pathway (43). Therefore, therapeutic tools, via targeting Rac1 and VASP interaction $(13,36,37)$, represent a promising drug candidate for cancer treatment.

In conclusion, our study demonstrates that VASP regulates cell migration and metastasis in osteosarcoma. Furthermore, VASP is a downstream effector of Rac1 and thus may serve as a potential drug target for osteosarcoma treatment.

\section{References}

1. Witlox MA, Lamfers ML, Wuisman PI, Curiel DT and Siegal GP: Evolving gene therapy approaches for osteosarcoma using viral vectors: review. Bone 40: 797-812, 2007.

2. Grimer RJ, Taminiau AM and Cannon SR: Surgical outcomes in osteosarcoma. J Bone Joint Surg Br 84: 395-400, 2002.

3. Revenu C, Athman R, Robine S and Louvard D: The co-workers of actin filaments: from cell structures to signals. Nat Rev Mol Cell Biol 5: 635-646, 2004.

4. Scott JA, Shewan AM, Den Elzen NR, Loureiro JJ and Gertler FB: Ena/VASP proteins can regulate distinct modes of actin organization at cadherin-adhesive contacts. Mol Biol Cell 17: 1085-1095, 2006.

5. Koretzky GA, Abtahian F, Derimanov GS, et al: Regulation of hematopoietic cell development and activation by adapter proteins. Immunol Res 27: 357-366, 2003.

6. Kragtorp KA and Miller JR: Regulation of somitogenesis by Ena/VASP proteins and FAK during Xenopus development. Development 133: 685-695, 2006.

7. Massberg S, Gruner S, Konrad I, et al: Enhanced in vivo platelet adhesion in vasodilator-stimulated phosphoprotein (VASP)deficient mice. Blood 103: 136-142, 2004.

8. Dertsiz L, Ozbilim G, Kayisli Y, Gokhan G, Demircan A and Kayisli U: Differential expression of VASP in normal lung tissue and lung adenocarcinomas. Thorax 60: 576-581, 2005.

9. Wang Y, Dong H, Zhu M, et al: Icariin exterts negative effects on human gastric cancer cell invasion and migration by vasodilatorstimulated phosphoprotein via Rac1 pathway. Eur J Pharmacol 635: 40-48, 2010.

10. Bonello L, Camoin-Jau L, Armero S, et al: Tailored clopidogrel loading dose according to platelet reactivity monitoring to prevent acute and subacute stent thrombosis. Am J Cardiol 103: 5-10, 2009.

11. Aszódi A, Pfeifer A, Ahmad M, et al: The vasodilator-stimulated phosphoprotein (VASP) is involved in cGMP-and cAMPmediated inhibition of agonist-induced platelet aggregation, but is dispensable for smooth muscle function. EMBO J 18: 37-48, 1999.

12. Hohenstein B, Kasperek L, Kobelt DJ, et al: Vasodilatorstimulated phosphoprotein-deficient mice demonstrate increased platelet activation but improved renal endothelial preservation and regeneration in passive nephrotoxic nephritis. J Am Soc Nephrol 16: 986-996, 2005.

13. Han G, Fan B, Zhang Y, et al: Positive regulation of migration and invasion by vasodilator-stimulated phosphoprotein via Rac1 pathway in human breast cancer cells. Oncol Rep 20: 929-939, 2008.

14. Bae YH, Ding Z, Zou L, Wells A, Gertler F and Roy P: Loss of profilin 1 expression enhances breast cancer cell motility by Ena/ VASP proteins. J Cell Physiol 219: 354-364, 2009.

15. Bear JE, Loureiro JJ, Libova I, Fässler R, Wehland J and Gertler FB: Negative regulation of fibroblast motility by Ena/ VASP proteins. Cell 101: 717-728, 2000.

16. Bear JE, Svitkina TM, Krause M, et al: Antagonism between Ena/VASP proteins and actin filament capping regulates fibroblast motility. Cell 109: 509-521, 2002.

17. Ridley AJ, Paterson HF, Johnston CL, Diekmann D and Hall A: The small GTP-binding protein rac regulates growth factorinduced membrane ruffling. Cell 70: 401-410, 1992.

18. Pankov R, Endo Y, Even-Ram S, et al: A Rac switch regulates random versus directionally persistent cell migration. J Cell Biol 170: 793-802, 2005.

19. del Pozo MA, Alderson NB, Kiosses WB, Chiang $\mathrm{HH}$, Anderson RGW and Schwartz MA: Integrins regulate Rac targeting by internalization of membrane domains. Science 303 : 839-842, 2004. 
20. Carabeo RA, Dooley CA, Grieshaber SS and Hackstadt T: Rac interacts with Abi 1 and WAVE2 to promote an Arp2/3 dependent actin recruitment during chlamydial invasion. Cell Microbiol 9: 2278-2288, 2007.

21. Heremans H, Billiau A, Cassiman JJ, Mulier J and De Somer P: In vitro cultivation of human tumor tissues. II. Morphological and virological characterization of three cell lines. Oncology 35 246-252, 1978

22. Tzanakakis G, Nikitovic D, Katonis P, Kanakis I and Karamanos N: Expression and distribution of $\mathrm{N}$ acetyl and $\mathrm{N}$ glycolylneuraminic acids in secreted and cell associated glycoconjugates by two human osteosarcoma cell lines. Biomed Chromatogr 21: 406-409, 2007.

23. Hu X, Yu AX, Qi BW, et al: The expression and significance of IDH1 and p53 in osteosarcoma. J Exp Clin Cancer Res 29: 43, 2010.

24. Scanlon E and Murthy S: The process of metastasis. CA Cancer J Clin 41: 301-305, 1991.

25. Endo-Munoz L, Cumming A, Rickwood D, et al: Loss of osteoclasts contributes to development of osteosarcoma pulmonary metastases. Cancer Res 70: 7063-7072, 2010.

26. Egeblad M and Werb Z: New functions for the matrix metalloproteinases in cancer progression. Nat Rev Cancer 2: 161-174, 2002.

27. Barzik M, Kotova TI, Higgs HN, et al: Ena/VASP proteins enhance actin polymerization in the presence of barbed end capping proteins. J Biol Chem 280: 28653-28662, 2005.

28. Bear JE and Gertler FB: Ena/VASP: towards resolving a pointed controversy at the barbed end. J Cell Sci 122: 1947-1953, 2009.

29. Rottner K, Behrendt B, Small JV and Wehland J: VASP dynamics during lamellipodia protrusion. Nat Cell Biol 1: 321-322, 1999.

30. Lebrand C, Dent EW, Strasser GA, et al: Critical role of Ena/ VASP proteins for filopodia formation in neurons and in function downstream of netrin-1. Neuron 42: 37-49, 2004.

31. Akin O and Mullins RD: Capping protein increases the rate of actin-based motility by promoting filament nucleation by the Arp2/3 complex. Cell 133: 841-851, 2008.

32. Ferron F, Rebowski G, Lee SH and Dominguez R: Structural basis for the recruitment of profilin-actin complexes during filament elongation by Ena/VASP. EMBO J 26: 4597-4606, 2007.
33. Laurent V, Loisel TP, Harbeck B, et al: Role of proteins of the Ena/VASP family in actin-based motility of Listeria monocytogenes. J Cell Biol 144: 1245-1258, 1999.

34. Loisel TP, Boujemaa R, Pantaloni D and Carlier MF: Reconstitution of actin-based motility of Listeria and Shigella using pure proteins. Nature 401: 613-616, 1999.

35. Coppolino MG, Krause M, Hagendorff P, et al: Evidence for a molecular complex consisting of Fyb/SLAP, SLP-76, Nck, VASP and WASP that links the actin cytoskeleton to Fcgamma receptor signalling during phagocytosis. J Cell Sci 114: 4307-4318, 2001.

36. Zhang Y, Han G, Fan B, et al: Green tea (-)-epigallocatechin3-gallate down-regulates VASP expression and inhibits breast cancer cell migration and invasion by attenuating Racl activity. Eur J Pharmacol 606: 172-179, 2009.

37. Zhang L, Wang T, Wen X, et al: Effect of matrine on HeLa cell adhesion and migration. Eur J Pharmacol 563: 69-76, 2007.

38. Lanier LM, Gates MA, Witke W, et al: Mena is required for neurulation and commissure formation. Neuron 22: 313-325, 1999.

39. Lauffenburger DA and Horwitz AF: Cell migration: a physically integrated molecular process. Cell 84: 359-369, 1996.

40. Chen L, Liao G, Waclaw RR, et al: Rac1 controls the formation of midline commissures and the competency of tangential migration in ventral telencephalic neurons. J Neurosci 27 : 3884-3893, 2007.

41. Pasic L, Kotova T and Schafer DA: Ena/VASP proteins capture actin filament barbed ends. J Biol Chem 283: 9814-9819, 2008.

42. Fryer BH, Wang C, Vedantam S, et al: cGMP-dependent protein kinase phosphorylates p21-activated kinase (Pak) 1, inhibiting Pak/Nck binding and stimulating Pak/vasodilator-stimulated phosphoprotein association. J Biol Chem 281: 11487-11495, 2006.

43. Lohmann SM and Walter U: Tracking functions of cGMPdependent protein kinases (cGK). Front Biosci 10: 1313-1328, 2005. 